NEUES AUS ITAS

\section{ITAS legt "Blütenträume" vor}

\author{
von Knud Böhle, ITAS
}

Die Kommerzialisierung des Internet, die einhergeht mit der Entwicklung des InternetHandels und der Entstehung von Zahlungssystemen für das Internet, bildete den Hintergrund für die vom BMBF in Auftrag gegebene Technikfolgenabschätzung zu elektronischen Zahlungssystemen für digitale Produkte und Dienstleistungen im Internet. Das Projekt wurde im Dezember 1998 abgeschlossen. Das Stichwort "Blütenträume" im Titel des Abschlußberichts ist weniger auf die Träume von Geldfälschern gemünzt, als vielmehr auf die vielerorts einkehrende Desillusionierung, daß unter der quirligen Oberfläche täglich wechselnder Neuheiten der Innovationsprozeß in der Breite nur langsam vorankommt und daß insbesondere der Traum vom "elektronischen Bargeld" als Zahlungsmittel im Internet auf unbestimmte Zeit noch ein Leitbild ohne Lobby bleiben wird.

\section{Zum Projekt Elektronische Zahlungssyste- me im Internet (PEZ)}

In dem Projekt wurde eine problemorientierte Sachstandsanalyse erarbeitet, die den Bedarf an neuen elektronischen Zahlungssystemen anhand der Praxiserfordernisse des Internet-Handels bemißt und gleichzeitig die Interessen der Akteure (Handel, Verbraucher, Kreditwirtschaft, Technologiefirmen, Gesetzgeber) als treibende oder retardierende Kräfte sowie den Strukturwandel der Branchen in diesem Innovationsprozeß zu identifizieren suchte. Die wichtigsten Informationsquellen waren Expertengespräche mit mehr als 60 Personen und die (auch teilnehmende) Beobachtung des sich im Internet entwickelnden Geschäftsverkehrs.

Vom Gegenstand her untersuchte das Projekt insbesondere den Teil des Internet-Handels, der über den klassischen Versandhandel mit elektronischen Bestellwegen hinausgeht: digitale Produkte, Dienstleistungen und Anrechte
(Eintrittskarten u.ä.). Bei der Untersuchung der Zahlungssysteminnovationen kam es dagegen darauf an, den Fokus nicht zu eng zu wählen. Deshalb wurde nicht nur das stark diskutierte Thema "elektronisches Geld" (Cybermoney, Cybercash etc.) aufgegriffen, sondern auch das Potential aller weiteren unbaren Zahlungsverfahren für den Zahlungsverkehr im Internet ausgelotet.

\section{Hauptergebnisse in fünf Thesen}

1. Der Erfolg des Internet-Handels hängt nicht von neuartigen Zahlungssystemem ab

In der Studie wird gegen die weit verbreitete Annahme argumentiert, der Erfolg des InternetHandels hinge gegenwärtig von neuartigen elektronischen Zahlungssystemen ab. Insofern physische Güter über das Internet nur bestellt werden, taugen auch die altbekannten Verfahren, die im Versandhandel gebräuchlich sind, uneingeschränkt. Dazu kommt, daß im Zuge der technischen Modernisierung im Bankensektor die meisten herkömmlichen Bezahlverfahren (Überweisung, Lastschrift, Kreditkartenzahlung u.a.) bereits in das Internet "migrieren", d.h. im Internet direkt abgewickelt werden können. Vor allem Kreditkartenzahlungen - mit unterschiedlichen Sicherheitsniveaus allerdings - sind im Internet bereits gang und gäbe und ermöglichen auch grenzüberschreitende Zahlungen. Außerdem bieten viele Anbieter ihre Produkte und Dienste im Internet immer noch gratis an. Sie sind nicht auf eine direkte Bezahlung angewiesen und finanzieren sich z.B. durch Werbung oder setzen auf spätere Anschlußgeschäfte.

2. Eine zunehmend bedeutendere "Zahlungssystemlücke" gibt es im klein- und kleinstpreisigen Bereich

Bedarf an neuartigen, elektronischen Zahlungsverfahren entsteht vor allem im klein- und kleinstpreisigen Bereich. Zum einen ist es für viele kleinere Firmen und private Anbieter nicht möglich, an den elektronischen Zahlungssystemen der Kreditwirtschaft als Händler teilzunehmen (wegen Fixkosten, Gebühren, Vertragsbedingungen etc.). Zum anderen entstehen zunehmend automatisierte Dienstleistungen im 
Internet, die sich bei hohem Nutzungsaufkommen schon bei Einnahmen von Pfennigbruchteilen tragen würden. Im ersten Fall wären Zahlungsverfahren gefordert, die möglichst allgemein und gleichzeitig voraussetzungslos für jedermann wären, so daß auch spontane und sporadische Kaufakte im Internet stattfinden können. Im zweiten Fall wären Zahlungsverfahren gefordert, die auch kleinste Beträge noch so kostengünstig abrechnen, daß die Einnahmen des Anbieters nicht durch die Kosten des Zahlungssystems vollständig aufgezehrt werden.

\section{Elektronisches Geld kann (nur) ein Teil der Lösung sein}

Unter elektronischem Geld werden häufig Zahlungssysteme zusammengefaßt, die gegenüber den herkömmlichen unbaren Zahlungsverfahren geringere Transaktionskosten aufweisen und deshalb eher für Kleinbetragszahlungen in Frage kommen. Die Einführung dieser neuen Zahlungsverfahren hat schon am stationären "Point of Sale" mit Akzeptanzproblemen zu kämpfen und ihre Einführung - ob softwarebasiert, chipkartenbasiert oder kombiniert - im Internet befindet sich erst oder noch immer im Stadium der Feldversuche. Diesen Zahlungssystemen kommt dennoch mittelfristig eine größere Bedeutung bei Zug-um-Zug-Geschäften im Internet zu - vor allem auch dann, wenn die Kunden Wert auf anonyme Zahlungen legen. Diese Zahlungssysteme werden dagegen nicht das Problem der "Micropayments" lösen und sie befriedigen auch nur partiell die Nachfrage nach "elektronischem Bargeld".

\section{Die Realisierung "elektronischen Bar- gelds" liegt noch in der Zukunft}

Gemessen an den Eigenschaften des Bargeldes (Zirkulationsfähigkeit, Diskriminierungsfreiheit, Geltungsbereich, Erfüllungswirkung u.a.m.) sind die als elektronisches Geld angebotenen Verfahren noch weit vom Leitbild "elektronischen Bargelds" entfernt. Das liegt zum einen daran, daß die tatsächlich im Internet anzutreffenden Formen elektronischen Geldes heute vorwiegend nur "Einmal- bzw. Einwegzahlungen" (Kunde $\rightarrow$ Händler $\rightarrow$ Bank) erlauben, folglich eine Zirkulation von Geld (wie beim Bargeld) nicht oder in nur sehr engen
Grenzen vorkommt. Zum anderen fehlt dem heutigen elektronischen Geld der Infrastrukturcharakter des Bargelds. Die vorfindlichen Systeme sind nicht allgemein einsetzbar, ihre Zahlungseinheiten sind nicht zwischen den Systemen austauschbar und sie sind in ihrer Nutzung mit teilweise erheblichen Gebühren verbunden.

5. "Elektronisches Bargeld" ist eine politische Forderung

Die derzeitigen Regelungen reservieren den Banken das Monopol auf die Herausgabe von elektronischem Geld. Da die Interessen der Kreditwirtschaft aber nicht unbedingt mit dem Leitbild "elektronischen Bargelds" in Einklang zu bringen sind, wird es "elektronisches Bargeld" nur dann geben - die Lösung der Sicherheitsfragen vorausgesetzt -, wenn sich politisch wirksame Interessen daran knüpfen. Die Forderung der Verbraucherverbände, die Wahlfreiheit der Zahlungsmittel auch im Internet herzustellen, könnte "elektronisches Bargeld" mit einschließen. Ebenso könnte von der Politik aus ein Bedarf gesehen werden, auch im Internet ein allgemeines, vielleicht sogar gesetzliches Zahlungsmittel bereitzustellen. Dies bedeutet nicht automatisch, daß der Staat selbst die Herausgeberfunktion für "elektronisches Bargeld" zu übernehmen hätte. Aufgefordert ist die Politik jedoch in jedem Fall, die nötigen Rahmenbedingungen zu setzen.

\section{Weitere Themen des Projekts}

Ausführlicher werden in dem Bericht folgende Aspekte behandelt: der Platz des klassischen Versandhandels im Internet-Handel, der Handel mit digitalen Produkten, Dienstleistungen und Anrechten und die dabei auftretende Zahlungssystemlücke, die Bedeutung des Internet-Handels für lokale Märkte, relevante Zahlungssysteminnovationen in Deutschland, Lösungen für kleine und kleinste Beträge, Wirtschaftlichkeitsfragen, das Innovationsinteresse der Kreditwirtschaft, die Entwicklung der internetfähigen Zahlungssysteme aus einer technikgenetischen Perspektive, der Zusammenhang von elektronischem Geld und Geldordnung, Definitionen elektronischen Geldes, die nicht-technischen Aspekte von Sicherheit und Anonymität, 
Risiken der Verbraucher beim Internet-Handel und offene Forschungsfragen. Das Schlußkapitel bietet unter der Überschrift "Zahlungssysteminnovationen: Ergebnisse und offene Forschungsfragen" ein Destillat der im Projekt erarbeiteten Einschätzungen und einen Aufriß noch zu bearbeitender Themen.

\section{Anmerkung}

Der Bericht von Knud Böhle und Ulrich Riehm: "Blütenträume" - Über Zahlungssysteminnovationen und Internet-Handel in Deutschland. Karlsruhe 1998 (FZKA 6161) kann bei den Autoren bestellt werden oder in elektronischer Form von den WWW-Seiten des Projektes heruntergeladen werden. Die Projektseite http://www.itas.fzk.de/deu/projekt/pez.htm enthält weitere Informationen über das Projekt, weitere Publikationen und Informationen zu dem projektbegleitend herausgegebenem elektronischen Newsletter EZI-N und der Mailingliste EZI-L, die auch jetzt noch aktiv ist und allen Interessierten offen steht.

\section{Kontakt}

Knud Böhle

Forschungszentrum Karlsruhe

Institut für Technikfolgenabschätzung

und Systemanalyse (ITAS)

Postfach 36 40, D-76021 Karlsruhe

Tel.: + 49 (0) 7247/82-2989 oder -2500

Fax: + 49 (0) 7247/82-4806

E-mail: boehle@itas.fzk.de

WWW-Projektseite:

http://www.itas.fzk.de/deu/projekt/pez.htm

$\gg$

\section{Projekt zu "Integrative Tech- nology Assessment" für die Europäische Kommission ab- geschlossen}

\section{von Michael Rader, ITAS}

Mitte 1998 wurde der Abschlußbericht eines Projekts vorgelegt, das die Entwicklung und Erprobung von "integrative technology assessment" (ITA) als Instrument zur Orientierung von Forschungs- und Entwicklungsvorhaben zum Ziel hatte. Auftraggeber war die Generaldirektion XIII der Europäischen Kommission im Rahmen des Programms INNOVATION.
Hauptauftragnehmer war das Flämische Institut für Technologische Forschung (VITO) in Mol. Das ITAS war an diesem Projekt als Unterauftragnehmer beteiligt.

Der Begriff "integrative technology assessment" wurde geprägt, um damit die Integration von Aspekten der Technikfolgenabschätzung in Forschungs- und Entwicklungsprojekten zu bezeichnen. Die Integration von TA soll dazu dienen, unerwünschte Technikfolgen so rechtzeitig zu erkennen, daß sie bereits vor der praktischen Anwendung beseitigt oder zumindest stark abgemildert werden können. Positive Effekte sollen dabei möglichst optimal zur Geltung gebracht werden, um mit Hilfe der ITA eine bessere Wettbewerbsfähigkeit und Akzeptanz technischer Produkte zu erreichen. Dieser Zielvorstellung entsprechend, werden die meisten Ansätze in diesem Umfeld in der Industrie erprobt, entweder als Kooperationsprojekt zwischen der Industrie und akademischen Institutionen, oder als Eigenprojekt besonderer Einrichtungen der Industrie.

Nach den Vorstellungen des Auftragnehmers sollten ursprünglich einige Beispiel von praktischen Anwendungen unterschiedlicher Ansätze zur Integration von Aspekten von Technikfolgenabschätzung in Forschungs- und Entwicklungsprojekten mit dem Hauptziel der Entwicklung von Technik identifiziert und auf ihre Tauglichkeit als "best practice" untersucht werden. Bei der Suche nach solchen Beispielen stellte sich jedoch schnell heraus, daß es nur wenige Projekte gegeben hat, welche die gewünschten Voraussetzungen erfüllten.

Nach Absprache mit dem Auftraggeber wurde daraufhin das Ziel des Projekts neu bestimmt. Ziel war es nunmehr, ein Quellen- und Methodenhandbuch zusammenzustellen, das in verschiedenen Phasen von geförderten FuEVorhaben Verwendung finden soll:

- Bei der Formulierung des Projektantrags durch den Antragsteller, dem mit dem Handbuch praktische Hinweise für mögliche Auswirkungsbereiche, die Identifikation von Betroffenen etc. gegeben werden sollen;

- $\quad$ bei der Begutachtung durch Kommissionsbedienstete und externe Experten, die mit dem Handbuch bei der Entwicklung von Kriterien für die Notwendigkeit einer TAKomponente und bei der Beurteilung der 
Angemessenheit von Vorschlägen für die Durchführung von ITA unterstützt werden sollen;

- $\quad$ bei der Konzeption und Durchführung der "integrativen" TA im Rahmen des eigentlichen FuE-Vorhabens.

Nicht zuletzt mußte ein Kompromiß zwischen den Wünschen des Auftraggebers nach "Patentrezepten" und dem eher pragmatischen methodischen Ansatz nahezu aller TA-Forscher gefunden werden.

Das Ergebnis ist ein "Informationspaket" für "Integrative Technikfolgenabschätzung", das potentielle Benutzer durch sämtliche Stufen eines Forschungs- und Entwicklungsvorhabens begleitet. Es enthält Hinweise zur Berücksichtigung von TA-Aspekten in Projektanträgen, zur Identifikation möglicher Problemfelder und Betroffenengruppen, sowie zur Planung einer integrierten Technikfolgenabschätzung in einem Vorhaben, das in erster Linie zur Forschung und Entwicklung im Bereich Technik dient. Schließlich gibt es Abschnitte zur Organisation des TA-Verfahrens und zur Integration von Ergebnissen in das FuE-Vorhaben. Eine wichtige Aufgabe des Informationspakets ist die Sensibilisierung von Verantwortlichen für FuEVorhaben für die nicht-technischen Aspekte des Technikeinsatzes, d.h. insbesondere für Auswirkungen und Gestaltungsbedingungen in den Bereichen Soziales und Umwelt.

Da davon auszugehen ist, daß die meisten FuE-Verantwortlichen bei der Durchführung der beschriebenen Schritte die Unterstützung oder zumindest Beratung durch erfahrene TAFachleute benötigen, enthält das Informationspaket umfassende Adressenverzeichnisse und eine annotierte Bibliographie einiger der relevantesten Veröffentlichungen. Sowohl die Adressenverzeichnisse als auch die Bibliographie sind nach Technikbereichen und Auswirkungsbereichen untergliedert.

Eingeleitet wird das Informationspaket mit einigen wesentlichen begrifflichen Bestimmungen und Unterscheidungen, die es dem Benutzer ermöglichen sollen, die für seine Problemkonstellation angemessene Vorgehensweise zu entwickeln. An Hand eines Modellfalls aus dem Bereich der Informationstechnik wird illustriert, wie ein "ITA-Verfahren" konzipiert werden und ablaufen könnte.
Im Informationspaket enthalten sind eine Anleitung zur Analyse der Interessen verschiedener Gruppen, die an der Entwicklung von Technik beteiligt sind bzw. von ihrem Einsatz potentiell betroffen sind, sowie eine exemplarische Checkliste möglicher Auswirkungsfelder von Technik. Diese Checkliste wird ergänzt durch eine Auflistung der häufigsten bekannten Auswirkungsbereiche der gegenwärtig aktuellen Technologien.

Abgerundet wird das Informationspaket mit einer kurzen Übersicht der gängigsten Ansätze der Technikfolgenabschätzung, Hinweisen zur Auswahl einer geeigneten Methode und einer Beschreibung einiger Methoden und Techniken der TA, die für eine Verwendung im Kontext von Forschungs- und Entwicklungsprojekten in Frage kommen.

Zwar bietet das Informationspaket dem engagierten FuE-Beteiligten ausreichende Informationen, um die notwendigsten Arbeiten auch ohne sachkundige Unterstützung durchzuführen, doch ist davon auszugehen, daß dort, wo komplexere Auswirkungen erwartet werden, die zu einer erheblichen Revision des ursprünglichen Forschungs- und Entwicklungsplans führen könnten, die Hinzuziehung von TA-Fachleuten erforderlich ist.

\section{Kontakt}

Robert Berloznik

Project Manager Technology Assessment Flemish Institute for Technological Research (VITO)

Boeretang 200, B-2400 Mol, Belgium

Tel.: +32 1433 59 34; Fax: +32 14321185

E-mail: berloznr@vito.be

URL: http://www.vito.be

oder

Michael Rader

Forschungszentrum Karlsruhe

Institut für Technikfolgenabschätzung

und Systemanalyse (ITAS)

Postfach 3640, D-76021 Karlsruhe

Tel.: +49-7247/82 2505; Fax: +49-7247/82 4806

E-mail: rader@itas.fzk.de

URL: http://www.itas.fzk.de 